\title{
Herramientas de visualización en ingeniería eléctrica basadas en Microsoft EXCEL: aplicación práctica al Teorema de Ferraris
}

\author{
Manuel Alcázar-Ortega ${ }^{a}$, Lina Montuori ${ }^{b}$, David Ribó-Pérez ${ }^{a}$ y Carlos Álvarez-Bel ${ }^{a}$ \\ aDepartamento de Ingeniería Eléctrica, Universitat Politècnica de València, Camino de Vera, s/n, \\ edificio 5E, 2a planta. 46022 Valencia (España), malcazar@iie.upv.es, david.ribo@iie.upv.es, \\ calvarez@die.upv.es

\footnotetext{
${ }^{\mathrm{b}}$ Departamento de Termodinámica Aplicada, Universitat Politècnica de València, Camino de Vera, s/n, edificio 5J, $2^{\text {a }}$ planta. 46022 Valencia (España), lmontuori@upvnet.upv.es
}

\begin{abstract}
This paper illustrates the high potential of Microsoft EXCEL for the implementation of tools oriented to the visualization of fundamental principles in Electrical Engineering for a better students understanding. In particular, the application to the Ferraris' Theorem, which bases the operation of rotating electric machines, is considered and put into practice. Thus, the graphical representation of the phasor diagram of a rotating machine, is obtained, which allows students to visualize how the modification of different key parameters such as the magnitude and angle of currents, affects the obtained magnetic field in the air gap. The paper evidences the suitability of Microsoft EXCEL for this kind of applications, which show significant advantages to students when compared to other commercial software. Consequently, the work here presented evidences how a spreadsheet may be used so as to help students to better understand the foundations of electromagnetism and electrical engineering by means of visualization tools which may be easily implemented by professors or even by the students themselves
\end{abstract}

Keywords: visualization tool, electrical engineering, electric machines, Ferraris' theorem

\footnotetext{
Resumen

Este artículo ilustra el alto potencial de Microsoft EXCEL para la implementación de herramientas orientadas a la visualización de principios fundamentales en ingeniería eléctrica para una mejor comprensión por parte de los alumnos. En particular, se ha considerado y puesto en práctica el Teorema de Ferraris, en el cual se basa la operación de las máquinas eléctricas rotativas. De esta forma, se puede representar gráficamente de una forma sencilla el diagrama fasorial de una máquina giratoria, permitiendo a los alumnos visualizar cómo al modificar los diferentes parámetros clave de la máquina (como la magnitud o el ángulo de las corrientes que circulan por el devanado inductor) se ve afedtado el campo magnético obtenido en el entrehierro. El artículo evidencia la adecuación de Microsoft EXCEL para este tipo de aplicaciones, mostrando ventajas significativas para los
} 
Herramientas de visualización en ingeniería eléctrica basadas en Microsoft EXCEL: aplicación práctica al Teorema de Ferraris

estudiantes cuando se compara con otras herramientas comerciales. En consecuencia, el trabajo que aquí se presenta pone de manifiesto la manera en la que una hoja de cálculo puede utilizarse para ayudar a los estudiantes a comprender mejor los fundamentos del electromagnetismo y la ingeniería eléctrica a través de herramientas de visualización que pueden ser fácilmente implementadas por los profesores o por los mismos estudiantes.

Palabras clave: herramienta de visualización, ingeniería eléctrica, máquinas eléctricas, teorema de Ferraris

\section{Introducción}

Uno de los problemas que más frecuentemente encuentran los docentes de enseñanzas técnicas es la dificultad para hacer entender a los alumnos algunos conceptos abstractos que se encuentran en los fundamentos de muchas aplicaciones que encontramos en la vida cotidiana pero que no por ello resultan evidentes. En este contexto, la implementación y utilización de herramientas de visualización en el aula se ha convertido en una ayuda excepcional en las clases de materias científico-técnicas, lo que se ve favorecido por la aplicación de las tecnologías de información y comunicación (TICs) a la educación superior (Habib, 2017). Además, como han demostrado investigaciones pedagógicas previas (Gilbert, 2005), los alumnos obtienen un beneficio mucho mayor cuando, en lugar de utilizar herramientas o simuladores ya existentes, son ellos mismos los que crean sus propias herramientas de visualización. Por otra parte, el desarrollo de este tipo de herramientas en la ingeniería puede ayudar a trabajar algunas competencias transversales de una manera más sencilla comparada con otras metodologías clásicas (Gómez-Tejedora, et al., 2018). Además, las herramientas de visualización resultan de especial utilidad en cursos masivos on-line (MOOCs), donde se requiere que los estudiantes se responsabilicen de su propio aprendizaje (Kuosal, et al., 2016)

En la actualidad, se pueden encontrar ejemplos de herramientas de visualización para la representación de magnitudes relacionadas con los campos magnéticos, sean basadas en métodos de diferencias finitias (Elsherbeni, et al., 2000) o mediante integración numérica (Roldán-Blay \& Roldán-Porta, 2017). Por otro lado, dichas herramientas suelen estar compiladas mediante programas de simulación comerciales como ANSYS, MATLAB, VTK o PYTHON (Mohammadi, et al., 2008). Como alternativa, este artículo propone la utilización de Microsof EXCEL para este tipo de herramientas, ya que presenta múltiples ventajas frente a otros programas (Alcázar-Ortega \& Álvarez-Bel, 2018) como pueden ser:

- Su alto grado de implantación entre la comunidad universitaria, ya que los alumnos suelen estar familiarizados con su entorno aunque suela utilizarse más frecuentemente para otras tareas. Por lo tanto, el desarrollo de herramientas de visualización a través de este software ayuda a que los usuarios utilicen toda su potencialidad, habitualmente infrautilizada, sobre todo con lo referente al análisis con números complejos, cálculo vectorial y matricial y su interfaz gráfica. 
- Su fácil accesibilidad, ya que esta herramienta suele estar instalada en cualquier ordenador personal junto con el paquete de Microsoft Office. Esta característica hace posible que los alumnos puedan disponer de este software sin la necesidad de adquirir licencias específicas y puedan utilizarlo tanto en el ámbito académico como en sus propios equipos domésticos.

- Su idoneidad para la práctica docente, en tanto en cuanto son los alumnos quienes crean su propio programa mediante la introducción de las ecuaciones analíticas vistas en clase. Por su formato de hoja de cálculo, donde se muestran los resultados de todos los cálculos intermedios, EXCEL resulta idóneo para la detección de errores de una forma sencilla, permitiendo a los alumnos aprender de sus errores, lo cual afianza el proceso de aprendizaje (Sasso, 2015).

El artículo se estructura de la siguiente manera: El capítulo 2 presenta los objetivos de la aplicación descrita en este documento, los cuales se desarrollan de forma detallada en el capítulo 3, incluyendo el fundamento teórico y la implementación de la herramienta en Microsoft EXCEL. El capítulo 4 muestra los resultados obtenidos en un caso práctico, tanto para sistemas de corriente equilibrados como desequilibrados. Finalmente, las conclusiones del presente trabajo se incluyen en el capítulo 5 .

\section{Objetivos}

Los objetivos que persigue el trabajo que se presenta en este documento son los siguientes:

- En primer lugar, evidenciar la potencialidad del programa Microsoft EXCEL para la visualización de conceptos teóricos relacionados con el campo de la ingeniería y su aplicación práctica para una mejor comprensión por parte del alumnado.

- Describir una herramienta de visualización desarrollada por los autores con dicho software para ilustrar el Teorema de Ferraris, que justifica los principios básicos de funcionamiento de una máquina síncrona rotatoria y las transformaciones energéticas que tienen lugar en ella.

- Mostrar a los docentes, tanto en este campo como en otros campos ingenierísticos, el potencial de Microsoft EXCEL para el desarrollo de herramientas similares, así como sus ventajas frente a otros paquetes de software comerciales.

- Finalmente, facilitar la comprensión por parte del alumnado de conceptos abstractos más o menos complejos que pueden ilustrarse de forma práctica mediante sencillas aplicaciones programables en una hoja de cálculo.

Los dos primeros objetivos quedan alcanzados en este trabajo, si bien la validación de los dos últimos objetivos aquí planteados queda emplazada para futuras aplicaciones.

\section{Desarrollo de la innovación}

\subsection{Fundamento teórico}

El Teorema de Ferraris (Galileo Ferraris, 1847-1897) establece que cuando un sistema de tres corrientes eléctricas alternas de frecuencia $f$ alimenta un sistema de tres devanados fijos 
desfasados $120^{\circ}$ magnéticos en el espacio, se produce un campo magnético giratorio de módulo constante y con una frecuencia igual a la de las corrientes que circulan por los devanados (Fraile Mora, 1995). El Teorema de Ferraris, en el que se basa el funcionamiento de las máquinas eléctricas rotatorias, puede generalizarse a un sistema de $m$ fases con la misma pulsación $\omega$ (Serrano Iribarnegaray, 1989), aunque en la práctica, los sistemas trifásicos son los que realmente se utilizan en la industria.

El Teorema de Ferraris así formulado justificaría el funcionamiento de un motor eléctrico de corriente alterna: si en el seno del campo magnético rotatorio producido por el sistema de corrientes eléctricas se coloca un cuerpo magnético que, atraído por dicho campo, pueda girar libremente (un imán permanente o un electroimán), dicho cuerpo girará a la velocidad del campo magnético que lo arrastra. No obstante, de acuerdo con el principio de reversibilidad de las máquinas eléctricas, el Teorema de Ferraris podría formularse de forma inversa, con lo que se obtendría la justificación del funcionamiento de un generador eléctrico: si un campo magnético rotatorio de magnitud constante gira en el seno de un sistema formado por tres devanados fijos en el espacio y desfasados $120^{\circ}$ magnéticos en el espacio, se inducirá un sistema de tensiones alternas igualmente desfasadas $120^{\circ}$ en el tiempo con una frecuencia igual a la de la pulsación a la que gira el campo magnético en el interior de dicho sistema.

A continuación se justificará matemáticamente la aplicación del Teorema de Ferraris a la creación de un campo magnético rotatorio de modulo y velocidad constantes a partir de un sistema de tres corrientes desfasadas $120^{\circ}$ en el tiempo.

Considérese un cilindro hueco como el que se muestra en la Figura 1, a lo largo de cuya longitud se han dispuesto tres bobinas desfasadas entre sí 120 grados geométricos. Este cilindro hueco correspondería al estator de una máquina eléctrica rotatoria, en cuyo interior se colocaría la parte móvil o rotor.

La bobina $a a^{\prime}$ 'se ha dispuesto con orientación vertical, de acuerdo con la dirección indicada por el eje de ordenadas colocado en su interior para poder representar los fasores correspondientes al campo magnético producido por cada una de estas corrientes. Se considera que la corriente entra a la bobina por el terminal $a$ y sale por el terminal $a$ '.

La bobina $b b^{\prime}$ 'se representa desfasada $120^{\circ}$ con respecto la bobina $a a^{\prime}$ ' en sentido antihorario, siendo la dirección de la corriente análoga a la de la bobina $b b^{\prime}$ (entrando por $b$ y saliendo por $\left.b^{\prime}\right)$. De firma similar, la bobina $c c^{\prime}$ ' se ha colocado desfasada $120^{\circ}$ con respecto a la bobina $b b^{\prime}$ y $240^{\circ}\left(\mathrm{o}-120^{\circ}\right)$ con respecto a la bobina $a a^{\prime}$.

Considérese que cada una de las bobinas anteriores se alimenta con una corriente desfasada en el tiempo $120^{\circ}$ con respecto a cada una de las otras dos. Las ecuaciones de cada una de estas corrientes vendrían dadas por las siguientes expresiones:

$$
\begin{aligned}
& i_{a a^{\prime}}=\sqrt{2} \cdot I_{e f} \cdot \operatorname{sen}(\omega \cdot t) \quad \mathrm{A} \\
& i_{b b^{\prime}}=\sqrt{2} \cdot I_{e f} \cdot \operatorname{sen}\left(\omega \cdot t-120^{\circ}\right) \mathrm{A} \\
& i_{c c^{\prime}}=\sqrt{2} \cdot I_{e f} \cdot \operatorname{sen}\left(\omega \cdot t-240^{\circ}\right) \quad \mathrm{A}
\end{aligned}
$$


Donde $I_{e f}$ representa el valor eficaz de la corriente que circula por cada una de las bobinas; $t$ es el tiempo transcurrido; y $\omega$ es la pulsación de la onda de corriente, que se puede calcular a partir de la frecuencia de la corriente (en Europa, $50 \mathrm{~Hz}$ ) multiplicando por $2 \cdot \pi$ $(\omega=2 \cdot \pi \cdot f=100 \cdot \pi \mathrm{rad} / \mathrm{s})$.

De acuerdo con la Ley de Ampère (Chapman, 1993), como resultado de la corriente que circula por la bobina $a a^{\prime}$, aparecerá un campo magnético $\overrightarrow{B_{a a^{\prime}}}=\mu \cdot \overrightarrow{H_{a a^{\prime}}}$ en la dirección perpendicular al eje de dicha bobina y, de acuerdo con la regla de la mano derecha, con el sentido indicado en la Figura 1.

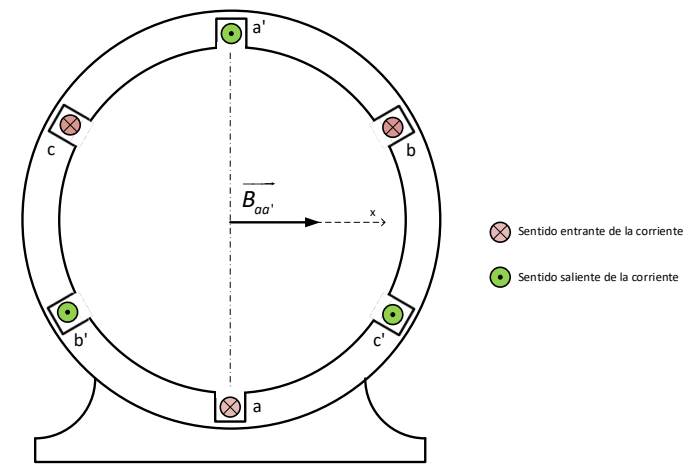

Figura 1. Campo magnético creado por la corriente que circula por la bobina aa'

Si se toma dicha dirección en el espacio como ángulo origen de referencias $\left(\varphi=0^{\circ}\right)$, el fasor espacial asociado (Serrano Iribarnegaray, 1989) a dicho campo magnético vendrá dado por la siguiente expresión:

$$
\overrightarrow{B_{a a^{\prime}}}=B_{\max } \cdot \operatorname{sen}(\omega \cdot t) \angle 0^{\circ} \mathrm{T}
$$

De forma similar, los fasores de campo magnético creado por las corrientes $i_{b b}$, e $i_{c c}$, serían los siguientes:

$$
\begin{aligned}
& \overrightarrow{B_{b b^{\prime}}}=B_{\text {max }} \cdot \operatorname{sen}\left(\omega \cdot t-120^{\circ}\right) \angle 120^{\circ} \mathrm{T} \\
& \overrightarrow{B_{c c^{\prime}}}=B_{\text {máx }} \cdot \operatorname{sen}\left(\omega \cdot t-240^{\circ}\right) \angle 240^{\circ} \mathrm{T}
\end{aligned}
$$

El campo magnético resultante en el interior del cilindro vendrá dado por la suma vectorial de los campos creados por cada una de las corrientes que circulan por cada bobina:

$$
\overrightarrow{B_{\text {Total }}}=\overrightarrow{B_{a a^{\prime}}}+\overrightarrow{B_{b b^{\prime}}}+\overrightarrow{B_{c c^{\prime}}} \mathrm{T}
$$

Para ver cómo evoluciona dicho campo magnético resultante a lo largo del tiempo, pueden darse valores a la variable $t$ que representa al tiempo. Por ejemplo, en el instante inicial $(t=0 \mathrm{~s})$, el campo magnético producido en cada bobina, y expresando el campo magnético máximo en tanto por uno $\left(B_{\max }=1 \mathrm{pu}\right)$, vendrá dado por las siguientes expresiones:

$$
\overrightarrow{B_{a a^{\prime}}}=\operatorname{sen}(0) \angle 0^{\circ}=0 \mathrm{pu}
$$


Herramientas de visualización en ingeniería eléctrica basadas en Microsoft EXCEL: aplicación práctica al Teorema de Ferraris

$$
\begin{aligned}
& \overrightarrow{B_{b b^{\prime}}}=\operatorname{sen}\left(0-120^{\circ}\right) \angle 120^{\circ}=-\frac{\sqrt{3}}{2} \angle 120^{\circ} \mathrm{pu} \\
& \overrightarrow{B_{c c^{\prime}}}=\operatorname{sen}\left(0-240^{\circ}\right) \angle 240^{\circ}=-\frac{\sqrt{3}}{2} \angle 240^{\circ} \mathrm{pu}
\end{aligned}
$$

Por tanto, el campo magnético resultante en el interior del cilindro será:

$$
\overrightarrow{B_{\text {Total }}}=0+\frac{-\sqrt{3}}{2} \angle 120^{\circ}+\frac{-\sqrt{3}}{2} \angle 240^{\circ}=1,5 \angle-90^{\circ} \mathrm{pu}
$$

Si el tiempo se incrementa en $5 \mathrm{~ms}(\omega \cdot t=0,5 \cdot \pi \mathrm{rad} / \mathrm{s})$, el campo magnético resultante en el interior del cilindro será:

$$
\overrightarrow{B_{\text {Total }}}=0+\frac{-1}{2} \angle 120^{\circ}+\frac{-1}{2} \angle 240^{\circ}=1,5 \angle 0^{\circ} \mathrm{pu}
$$

Es decir: transcurridos $5 \mathrm{~ms}$, el fasor de campo magnético ha girado en sentido antihorario un ángulo de $90^{\circ}$, permaneciendo constante el valor de su amplitud. De forma similar, cuando $t=10 \mathrm{~ms}$, el fasor de campo magnético se sitúa en un ángulo espacial de $180^{\circ}$, y en $t=20 \mathrm{~ms}$ (que corresponde con un período de la onda de corriente a $50 \mathrm{~Hz}$ ), el fasor habrá dado una vuelta completa al cilindro, tal y como se muestra en la Figura 2.
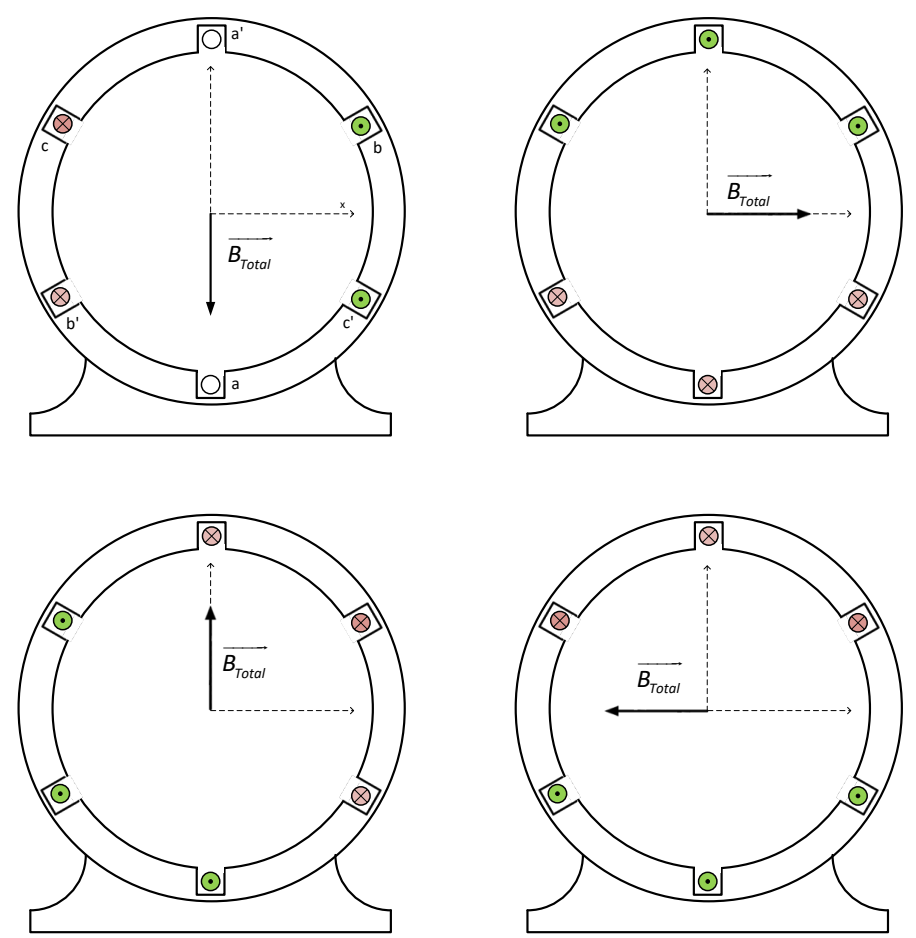

$\otimes$ $\odot$

Figura 2. Posición del fasor de campo magnético resultante para distintos valores de $t$ 
Por tanto, como queda ilustrado en este ejemplo, se concluye que el sistema de corrientes trifásicas desfasadas $120^{\circ}$ en el tiempo, da como resultado un campo magnético giratorio de magnitud constante igual a 1,5 veces el campo que produce una de las fases, el cual da una vuelta completa al cilindro en $20 \mathrm{~ms}$, que coincide con el período de la onda de corriente que alimenta cada una de las bobinas.

\subsection{Implementación de la herramienta de visualización con Microsoft EXCEL}

En esta sección se presenta la herramienta de visualización desarrollada en Microsoft EXCEL que ilustra de forma sencilla la aplicación del Teorema de Ferraris, expuesto en el apartado anterior. Para ello, se han utilizado dos hojas de cálculo dentro del mismo libro: la primera (“datos”) se utilizará para escribir la formulación matemática que permitirá obtener los resultados numéricos deseados; en la segunda hoja ("gráficos"), se implementarán los gráficos necesarios, los controles para interaccionar con la herramienta y las celdas con las variables de entrada que el usuario puede modificar para diseñar diferentes escenarios.

La herramienta se está utilizando desde hace cuatro años en las asignaturas "Generación, Transporte y Distribución de energía eléctrica" (con grupos de unos 50 alumnos) y "Sistemas Eléctricos de Potencia (con 15 alumnos de media por curso), ambas correspondientes al Máster en Ingeniería Industrial impartido por la Escuela Técnica Superior de Ingenieros Industirales de la Universidad Politécnica de Valencia. Como resultado, los alumnos han manifestado en numerosas ocasiones al profesorado cómo la utilización de esta herramienta les ha facilitado la comprensión de estos conceptos, los cuales, desde un punto de vista puramente teórico, pueden resultar demasiado abstractos para su fácil asimilación.

\subsubsection{Datos de entrada}

Los datos de entrada que el usuario puede modificar son los siguientes:

- Las corrientes que circularán por cada una de las bobinas. Es decir, que la herramienta permitirá visualizar el efecto de hacer circular por las tres bobinas la misma corriente o corrientes diferentes, con distintos ángulos temporales. Esta selección se realizará mediante un controlador tipo desplegable, que permitirá elegir la corriente que circula por cada bobina.

- El valor máximo del campo magnético producido por la corriente que circula por cada una de las tres bobinas. Por defecto, este valor será igual a 1 para todas las bobinas, pero su modificación permitirá visualizar el efecto de corrientes con diferente amplitud en el campo magnético resultante.

- El ángulo temporal de cada una de las corrientes que circula por las bobinas. De esta forma, la herramienta permitirá visualizar el efecto que tienen sobre el campo magnético sistemas desequilibrados de corriente. Por defecto, la herramienta fija inicialmente estos valores en $0,120^{\circ}$ y $240^{\circ}$ (régimen equilibrado).

- La frecuencia de la red. Por defecto, este valor se fijará a $50 \mathrm{~Hz}$, pero el simulador permite su modificación para visualizar el campo magnético producido en otros sistemas eléctricos (por ejemplo, en el sistema americano donde la frecuencia son $60 \mathrm{~Hz})$. 
- Finalmente, la última variable de entrada es el instante de tiempo para el que se desea obtener la visualización del campo magnético. Esta variable se establecerá mediante un contador, de forma que podrá incrementarse de forma automática mediante un botón de control ubicado en la hoja de cálculo de visualización del fichero de Microsoft EXCEL.

\subsubsection{Representación gráfica de la herramienta de visualización}

La herramienta de visualización mostrará los resultados de las simulaciones realizadas en dos gráficas diferentes:

- Gráfica 1: Fasor de campo magnético. Este gráfico representará el fasor espacial que indica el valor máximo del campo magnético en el instante de tiempo considerado, tanto para cada una de las bobinas como para el campo resultante. La representación obtenida sería similar a la mostrada en la Figura 2, para un instante de tiempo previamente seleccionado. El diagrama se ha colocado en el interior de una figura que representa la vista frontal del cilindro donde se encontrarían devanadas las tres bobinas consideradas en este sistema, el cual ha sido diseñado mediante el programa de Microsoft VISIO, siendo posteriormente exportado a la hoja de cálculo de Microsoft EXCEL.

- Gráfica 2: Representación de la onda de tensión magnética a lo largo del entrehierro. Este gráfico mostrará la onda de campo magnético proyectada a lo largo de la superficie desarrollada del cilindro, tanto para cada una de las bobinas como para el campo magnético resultante.

La representación se realizará mediante gráficos de dispersión con coordenadas X e Y. En el caso de la Gráfica 1, las coordenadas X e Y corresponderán a la parte real e imaginaria del fasor de campo magnético. En el caso de la Gráfica 2, la coordenada X representará el ángulo espacial, mientras que la coordenada $\mathrm{Y}$ corresponderá con la magnitud del vector de campo magnético.

\subsubsection{Cálculo del campo magnético en el entrehierro}

La evaluación matemática del campo magnético en el entrehierro se realizará parametrizando las expresiones (4), (5), (6) y (7) con el lenguaje de programación de Microsoft EXCEL. Para ello, el primer paso será colocar las variables de entrada descritas en la sección 3.2.1 en celdas de la hoja de cálculo. En particular, la posición de cada una de las variables se indica en la Tabla 1. Asimismo, el ángulo espacial de las bobinas se escribirá en otras tres celdas, tal y como se indica en la Tabla 2 pero, en este caso, el desfase se mantiene constante e igual a $120^{\circ}$ para cada par de bobinas.

Para obtener los datos de la Gráfica 1 (fasor espacial de campo magnético en el interior del cilindro) se calculará, en primer lugar, el fasor de campo magnético para cada una de las bobinas, que se escribirá en las celdas B12, B13 y B14. De acuerdo con (4) y considerando las variables indicadas en la Tabla 1, el campo generado por la corriente de la bobina aa' en el instante $t$, escrito en forma binómica en el lenguaje de Microsoft EXCEL, vendrá dado por la siguiente expresión: 
$=C O M P L E J O(B 1 * S E N O(2 *$ pi( $) * B 7 * B 8-R A D I A N E S(B 4)) * C O S(R A D I A N E S(B 9))$;

$B 1 * \operatorname{SENO}(2 *$ pi( $) * B 7 * B 8-R A D I A N E S(B 4)) * \operatorname{SENO}(\operatorname{RADIANES}(B 9)))$

Tabla 1. Variables de entrada de la herramienta de visualización

\begin{tabular}{|c|c|c|c|}
\hline Variable & Unidades & Símbolo & Celda \\
\hline $\begin{array}{l}\text { Valor máximo del campo magnético producido por la } \\
\text { corriente que circula por la bobina aa' }\end{array}$ & $\mathrm{pu}$ & $B_{a a^{\prime}}^{\text {máx }}$ & B1 \\
\hline $\begin{array}{l}\text { Valor máximo del campo magnético producido por la } \\
\text { corriente que circula por la bobina bb' }\end{array}$ & $\mathrm{pu}$ & $B_{b b^{\prime}}^{m a ́ x}$ & B2 \\
\hline $\begin{array}{l}\text { Valor máximo del campo magnético producido por la } \\
\text { corriente que circula por la bobina } \mathrm{cc}^{\prime}\end{array}$ & $\mathrm{pu}$ & $B_{c C^{\prime}}^{\operatorname{máx}}$ & B3 \\
\hline Desfase inicial de la corriente por la bobina aa' & grados & $\varphi_{a a^{\prime}}$ & B4 \\
\hline Desfase inicial de la corriente por la bobina bb' & grados & $\varphi_{b b^{\prime}}$ & B5 \\
\hline Desfase inicial de la corriente por la bobina $\mathrm{cc}^{\prime}$ & grados & $\varphi_{c c}{ }^{\prime}$ & B6 \\
\hline Frecuencia de la corriente & $\mathrm{Hz}$ & $f$ & B7 \\
\hline Tiempo de visualización & $\mathrm{s}$ & $t$ & B8 \\
\hline
\end{tabular}

Tabla 2. Variables de ángulo espacial entre las bobinas

\begin{tabular}{llccc}
\hline Variable & Unidades & Símbolo & Celda & Valor \\
\hline Ángulo espacial de la bobina aa' & grados & $\alpha_{a a^{\prime}}$ & $\mathrm{B} 9$ & 0 \\
Ángulo espacial de la bobina bb' & grados & $\alpha_{b b^{\prime}}$ & $\mathrm{B} 10$ & 120 \\
Ángulo espacial de la bobina cc' & grados & $\alpha_{c c^{\prime}}$ & $\mathrm{B} 11$ & 240 \\
\hline
\end{tabular}

De forma análoga, para las bobinas $b b^{\prime}$ y $c c^{\prime}$, la expresión del campo magnético será la siguiente:

$$
\begin{aligned}
& =\text { COMPLEJO }(B 2 * \operatorname{SENO}(2 * \text { pi( }) * B 7 * B 8-R A D I A N E S(B 5)) * C O S(R A D I A N E S(B 10)) \text {; } \\
& B 2 * \operatorname{SENO}(2 * \text { pi( }) * B 7 * B 8-R A D I A N E S(B 5)) * \operatorname{SENO}(\operatorname{RADIANES}(B 10))) \\
& =\operatorname{COMPLEJO}(B 3 * \operatorname{SENO}(2 * \text { pi }() * B 7 * B 8-R A D I A N E S(B 6)) * \operatorname{COS}(\operatorname{RADIANES}(B 11)) \text {; } \\
& B 3 * \operatorname{SENO}(2 * \text { pi() *B7*B8-RADIANES(B6))*SENO(RADIANES(B11)))}
\end{aligned}
$$

Calculado el campo asociado a cada bobina, el fasor resultante de campo total se obtendrá como la suma vectorial del producido en cada una de las fases. Para ello, se utilizará la función de suma de números complejos de Microsoft EXCEL. Este valor se escribirá en la celda B15:

$$
=I M \cdot S U M(B 12 ; B 13 ; B 14)
$$


Calculados los cuatro vectores de campo magnético (para las tres fases y el resultante), las coordenadas que permitirán representar gráficamente el fasor de campo serán, respectivamente, la parte real de cada vector de campo para abscisas y la parte imaginaria de cada vector para ordenadas.

Para obtener la representación de la Gráfica 2, será es necesario evaluar el valor que toma el campo magnético en función del ángulo espacial $\alpha_{e}$, desde 0 hasta $2 \pi$ radianes. Para ello, se calculará con Microsoft EXCEL el valor del campo magnético creado por la corriente que circula por cada bobina a lo largo de dicha circunferencia, en intervalos de $5^{\circ}$. El procedimiento de cálculo será el siguiente:

1. Escribir en una columna (por ejemplo columna D) el valor del ángulo espacial (a lo largo de la periferia del cilindro) en el que se va a evaluar el campo magnético, en intervalos de 5 grados (o 0,087 radianes). Ello dará un total de 72 valores (celdas D1 a D72)

2. En las tres columnas siguientes (E, F y G), se calculará el valor del campo magnético producido por la corriente que circula por cada bobina para cada valor de ángulo espacial. La expresión a escribir en la celda E1 para obtener el valor del campo asociado a la bobina aa' en la posición $\alpha_{e}=0$ será la siguiente:

$=A B S(\$ B \$ 12) * S E N O(A N G U L O(R A D I A N E S(\$ B \$ 12))+\operatorname{RADIANES}(\$ B \$ 9-D 1))$

$\mathrm{Al}$ arrastrar esta celda dentro de la columna $\mathrm{E}$ hasta la celda E72 se obtendrán los valores asociados a cada ángulo espacial de la columna D. Por tanto, la referencia D1 en (17) irá tomando valores sucesivos en cada fila para calcular el campo correspondiente a cada posición espacial indicada por el ángulo correspondiente.

De forma similar, el valor del campo magnético en la posición espacial $\alpha_{e}=0$ asociado a las bobinas $b b^{\prime}$ y $c c^{\prime}$ (celdas F1 y G1) se obtendrá con las siguientes expresiones:

$=A B S(\$ B \$ 13) * S E N O(A N G U L O(R A D I A N E S(\$ B \$ 13))+R A D I A N E S(\$ B \$ 10-D 1))$

$=A B S(\$ B \$ 14) * S E N O(A N G U L O(R A D I A N E S(\$ B \$ 14))+R A D I A N E S(\$ B \$ 11-D 1))$

3. El campo resultante en cada posición espacial $\alpha_{\mathrm{e}}$ será la suma del campo producido por cada bobina. En el caso de $\alpha_{e}=0$, el campo resultante será:

$=E 1+F 1+G 1$

\subsection{Contador para el incremento automático de tiempo}

De cara a facilitar la interacción del usuario con la herramienta de visualización, se puede programar de forma sencilla un contador de tiempo que incremente en intervalos previamente definidos el instante en el que se desea obtener la representación gráfica del campo.

De esta forma, la evolución temporal del campo magnético podría obtenerse fácilmente con un simple "click" a un botón ubicado junto al gráfico. Para ello es necesario insertar un control de formulario tipo botón y asignarle una subrutina (macro) donde se defina el funcionamiento del contador. Considerando un intervalo de $0,5 \mathrm{~ms}$ (es decir, que cada vez que se pulse el botón el tiempo se incrementará en $0,5 \mathrm{~ms}$ ), la subrutina asociada al botón de contador podría ser la mostrada en la Figura 3. 
Asimismo, otro controlador podría reinicializar fácilmente la simulación, simplemente escribiendo en la celda B8 el valor 0 al pulsar otro botón.

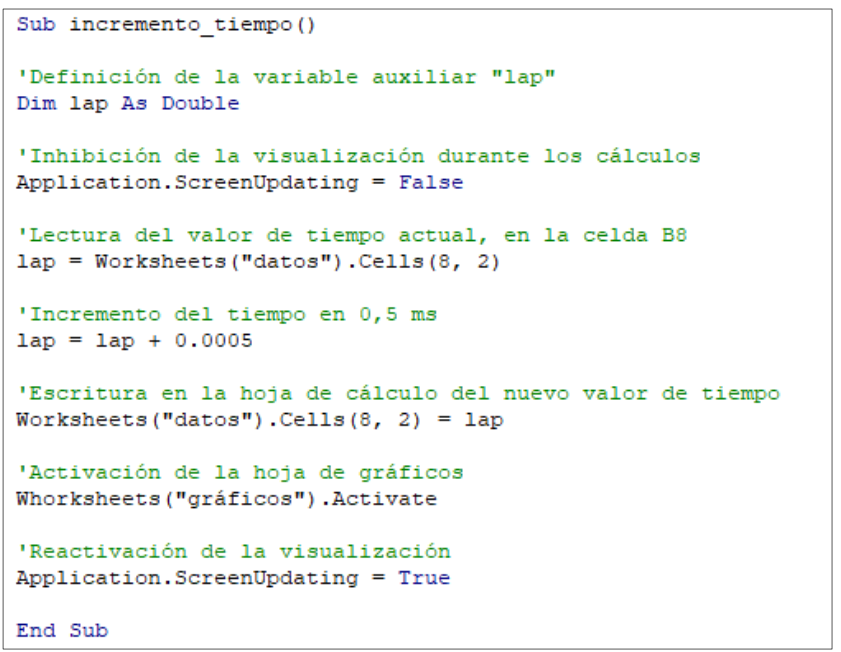

Figura 3. Macro para la programación del contador de tiempo automático

\subsection{Representación del afijo del campo}

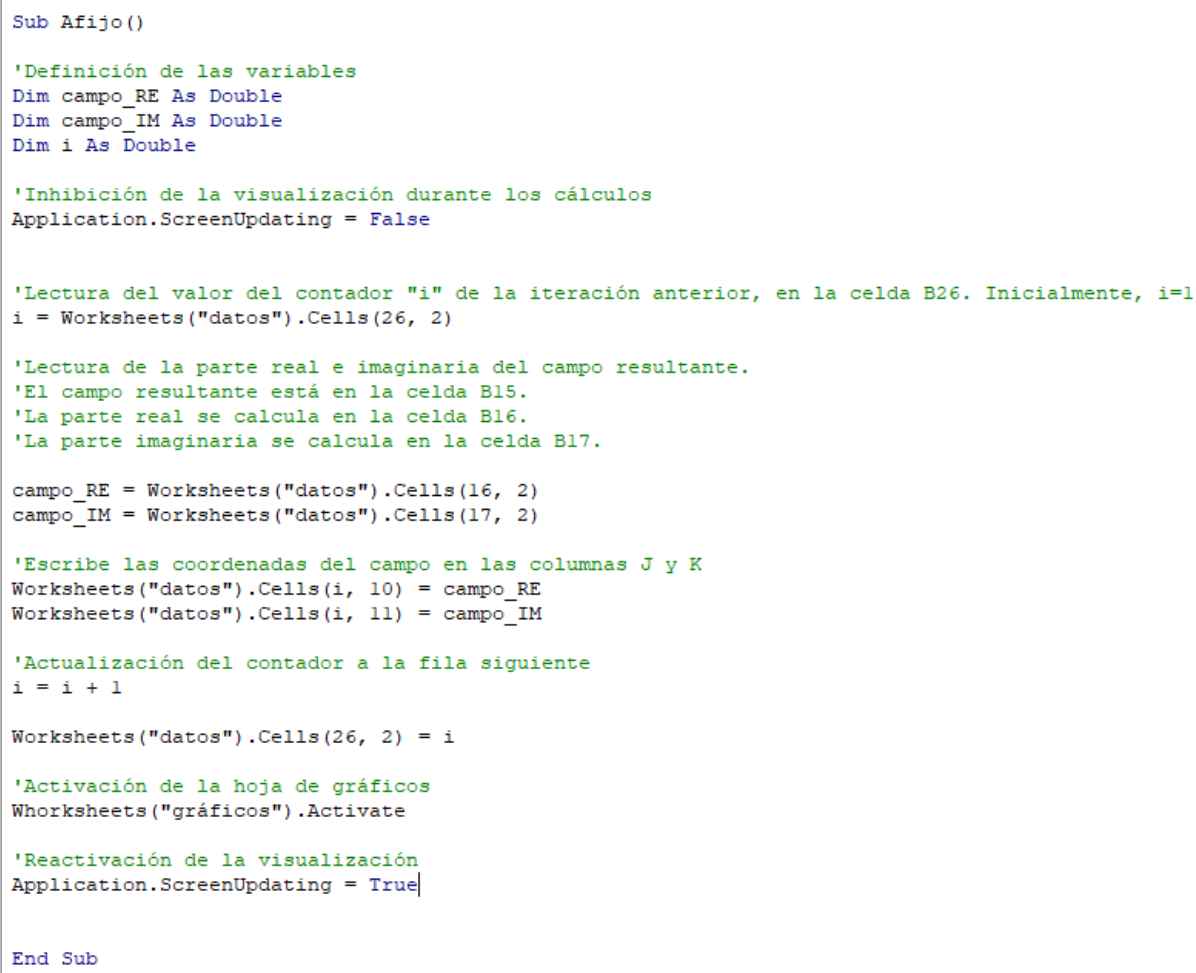

Figura 4. Macro para la programación del contador de tiempo automático 
Para identificar claramente la forma del campo magnético que aparece en el interior del cilindro, la herramienta de visualización contempla la representación gráfica del afijo que toma el vector de campo magnético a lo largo de una simulación para los diferentes intervalos de tiempo considerados. Es decir: cuando el fasor espacial de campo magnético resultante se mueve a lo largo del tiempo, deja un "rastro" que evidencia el tipo de campo creado por las corrientes seleccionadas (circular, elíptico o pulsante).

Para ello, se ha diseñado otra subrutina que guarda en la hoja "datos" la posición del afijo del fasor espacial de campo magnético hasta que se reinicie la simulación. Dicha subrutina se muestra en la Figura 4.

\section{Resultados}

A continuación, se muestran los resultados obtenidos con la herramienta de visualización que se presenta en este artículo para la representación del campo magnético producido en el interior de un cilindro por la corriente que circula por tres bobinas desfasadas $120^{\circ}$. Esta herramienta permite representar fácilmente el campo producido por cada corriente y por el conjunto del sistema con diferentes tipos de corriente senoidal en cada una de las bobinas. A modo de ejemplo, se mostrarán a continuación los campos obtenidos en el caso de alimentar las bobinas con corrientes de igual o distinta amplitud, y con un desfase igual o diferente de $120^{\circ}$. En otras palabras: la herramienta permite visualizar el campo producido tanto por sistemas equilibrados como por sistemas desequilibrados de corriente.

\subsection{Sistema equilibrado (campo giratorio circular)}

Cuando se alimentan las bobinas colocadas en el cilindro tal y como indica el teorema de Ferraris (misma amplitud y desfasadas $120^{\circ}$ en el tiempo), la herramienta de visualización muestra los diagramas que aparecen en la Figura 5 para los instantes $t=0, t=5 \mathrm{~ms}$ y $t=15 \mathrm{~ms}$. Tal y como se ha discutido anteriormente, la herramienta de visualización permite obtener el fasor de campo magnético (lado izquierdo de la figura) y la onda de campo en el entrehierro (lado derecho). Como se puede observar, el cambo resultante es giratorio (en sentido antihorario) y circular, de la misma forma que la onda de campo magnético se desplaza hacia la izquierda. Por otro lado, el lado de la bobina por el que entra o sale la corriente aparece indicado con un círculo verde en el caso del terminal por el que sale la corriente, o rojo con un aspa blanca en el caso del sentido entrante de la corriente. Dicha representación puede realizarse fácilmente con la opción "formato condicional" de Microsoft EXCEL, eligiendo una opción u otra en función de que la corriente en ese instante en ese punto sea positiva o negativa. 

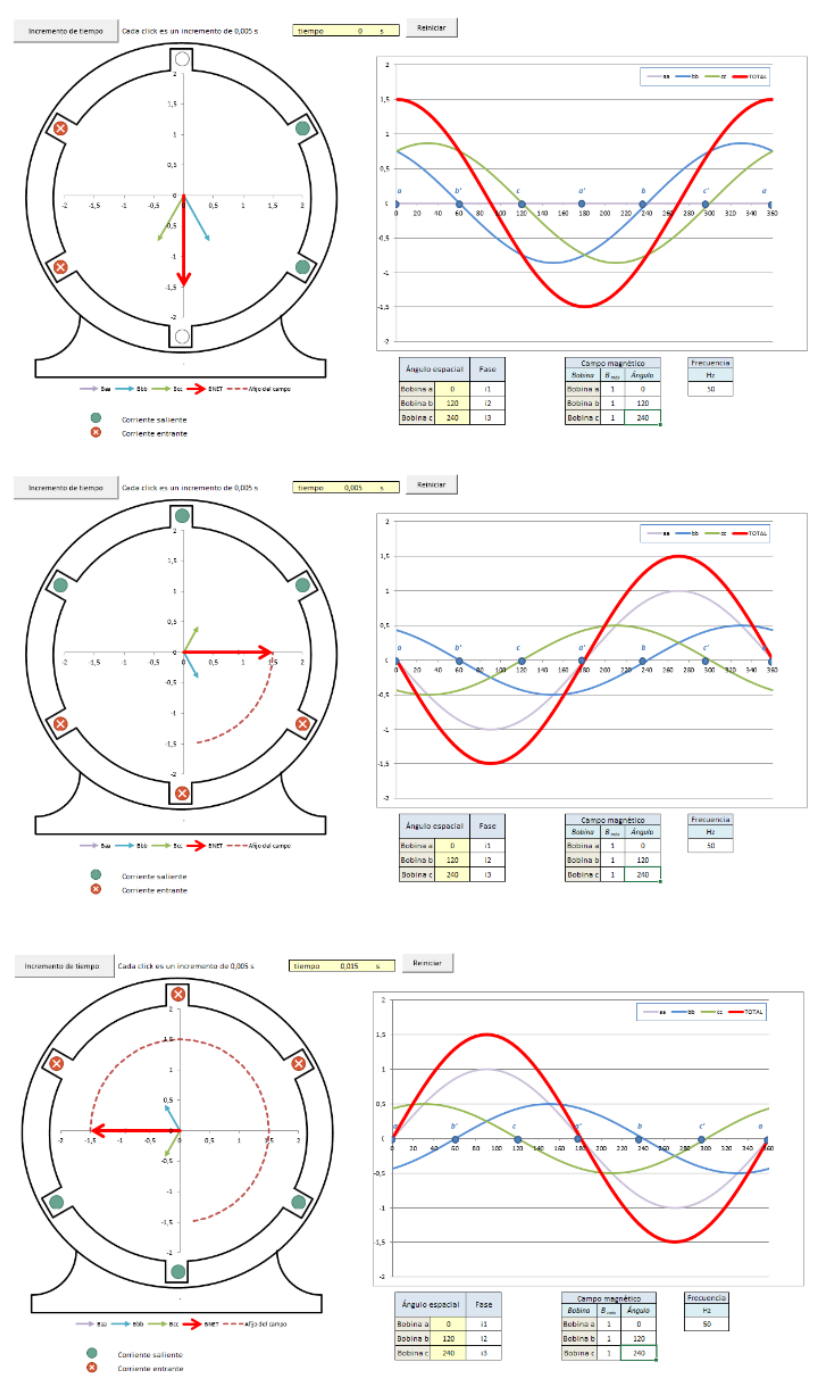

Figura 5. Pantallas de la herramienta de visualización para un sistema equilibrado de corrientes

\subsection{Sistema desequilibrado}

La herramienta de visualización también permite obtener la forma del campo magnético resultante en el caso de sistemas desequilibrados de corriente. A modo de ejemplo, se ilustra el caso de un sistema alimentado por las siguientes corrientes:

- Corriente de la bobina $a a^{\prime}$ : Amplitud de 1 pu y desfase inicial de $0^{\circ}$

- Corriente de la bobina $b b^{\prime}$ : Amplitud de 0,8 pu y desfase inicial $100^{\circ}$

- Corriente de la bobina $c c^{\prime}$ : Amplitud de 0,5 pu y desfase inicial de $280^{\circ}$

El resultado obtenido se muestra en la Figura 6, donde aparece un campo magnético giratorio pero de forma elíptica. 

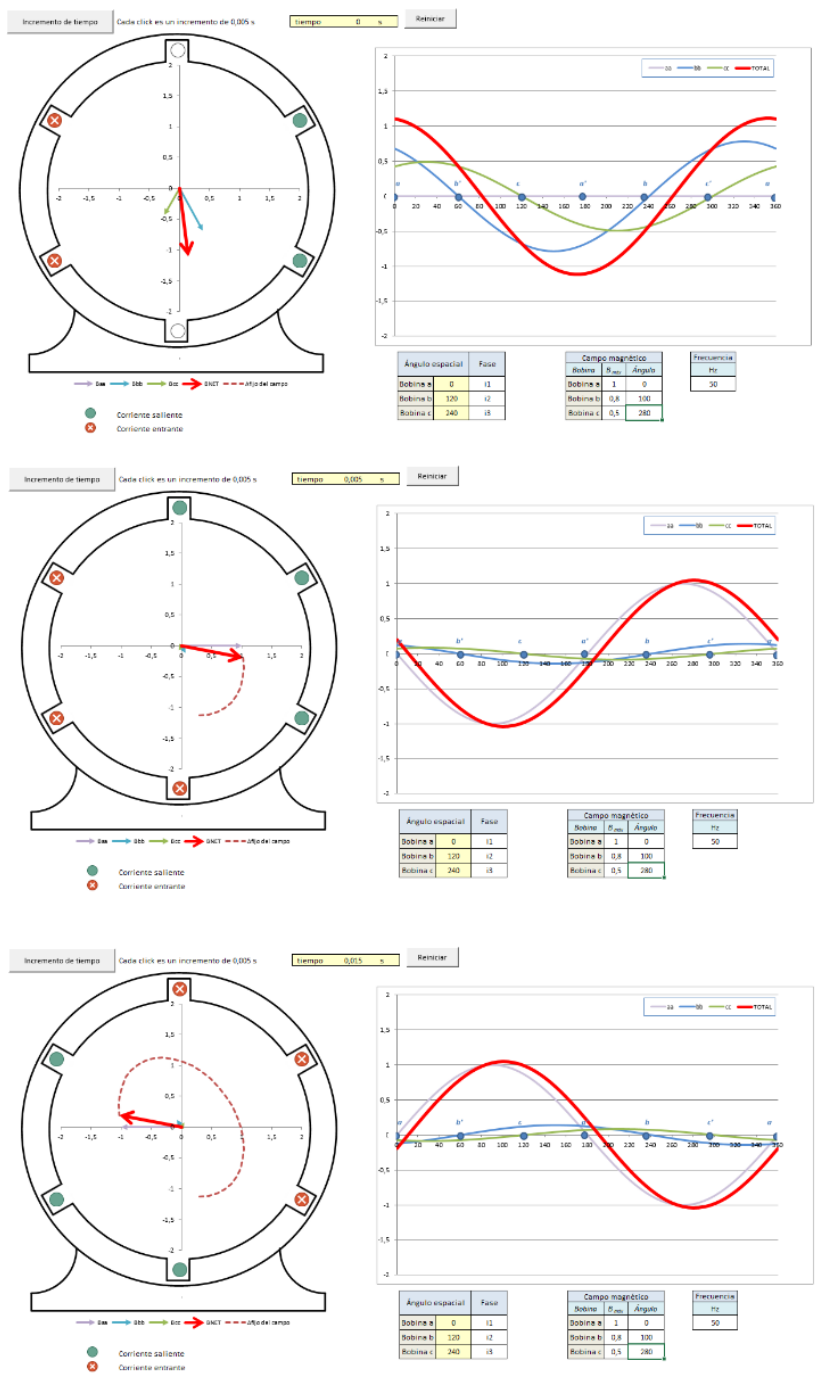

Figura 6. Pantallas de la herramienta de visualización para un sistema desequilibrado de corrientes

\section{Conclusiones}

Este artículo evidencia el potencial de una herramienta como Microsoft EXCEL para la visualización de conceptos abstractos de ingeniería como el campo magnético mediante el cálculo de números complejos y la representación gráfica de sistemas vectoriales y fasoriales. En particular, se han obtenido diferentes visualizaciones del campo magnético producido en el interior de un cilindro por un sistema de corrientes eléctricas desfasadas en el espacio y en el tiempo, lo que constituye la demostración del Teorema de Ferraris en el que se basa el funcionamiento de las máquinas eléctricas rotatorias.

Desde el punto de vista docente y educativo, la conveniencia de utilizar herramientas como Microsoft EXCEL para aplicaciones más o menos complejas, como la aquí presentada, queda justificada por varias razones. Algunas serían su fácil accesibilidad por parte de estudiantes 
y profesores, su alto grado de implantación a nivel usuario y su interfaz gráfica para la representación de resultados numéricos de una manera cómoda, sencilla y muy visual. Por otro lado, Microsoft EXCEL pone a disposición de los usuarios la posibilidad de realizar cálculos vectoriales, matriciales y de números complejos, lo cual, pese a ser un aspecto bastante desconocido por parte de los usuarios habituales de esta herramienta, la convierte en un entorno idóneo para la obtención y visualización de resultados prácticos en el campo de la ingeniería.

\section{Referencias}

Alcázar-Ortega, M. \& Álvarez-Bel, C., 2018. Utilización de Microsoft EXCEL en la enseñanza de sistemas eléctricos de potencia: desarrollo de un método matricial para la resolución del problema de despacho económico. IV Congreso Nacional de Innovación Educativa y Docencia en Red (IN-RED 2018), Volumen 1, pp. 1077-1090.

Chapman, S. J., 1993. Máquinas Eléctricas (ISBN 0070109141). Santa Fe de Bogotá: McGraw Hill Interamericana, S.A..

Elsherbeni, A. Z., Glisson, A. W., Riley, C. L. \& Smith, C. E., 2000. Tools for electromagnetic modeling and visualization using the FDTD Technique. Symposium on Antenna Technology and Applied Electromagnetics [ANTEM 2000].

Fraile Mora, J., 1995. Máquinas Eléctricas (ISBN 8474931436). Madrid: Servicio de Publicaciones. Colegio de Ingenieros de Caminos, Canales y Puertos de Madrid..

Gilbert, J. K., 2005. Visualization in Science Education. Dordrecht: Springer.

Gómez-Tejedora, J. A. y otros, 2018. Diseño y evaluación de un laboratorio virtual para visualizar momentos de un vector deslizante en 3D. IV Congreso Nacional de Innovación Educativa y Docencia en Red (IN-RED 2018), Volumen 1, pp. 299-312.

Habib, H., 2017. Role of ICT in Higher Education. International Journal of Creative Research Thoughts, 5(4), pp. 2810-2813.

Kuosal, K. y otros, 2016. Interactive Visualization Tools to improve learning and teaching in online learning environments. International Journal of Distance Education Technologies, 14(1), pp. 1-21.

Mohammadi, S. M. y otros, 2008. Interactive visualization of new electromagnetic quantities. SIGRAD. Estocolmo (Suecia), Volumen 1, pp. 71-74.

Roldán-Blay, C. \& Roldán-Porta, C., 2017. La medida del campo magnético y su cálculo y representación con CRMag. Valencia: Universitat Politècnica de València.

Sasso, P., 2015. El error como herramienta de aprendizaje. Reflexión Académica en Diseño y Comunicación, Volumen 25, pp. 25-27.

Serrano Iribarnegaray, L., 1989. Fundamentos de máquinas eléctricas rotativas (ISBN 8426707637). Barcelona: Marcombo Boixareu. 